\title{
The Ups and Downs of Non-Insulin Therapies: The Agony of Choice
}

The last decade has seen a rise in the number of new classes of non-insulin antihyperglycemic pharmacological therapies for type 2 diabetes, following a dearth of pharmacological developments. The "ups" include the ability of physicians to have an ever growing armamentarium of medications to use. However, coincident with this increase comes about confusion as to which medications might be most suitable for a particular patient. This confusion might be better described as the agony of choice, an inadvertent "down".

All anti-hyperglycemic medications lower glucose levels and glycated hemoglobin $(\mathrm{HbA} 1 \mathrm{c})$ to variable degrees, a feature necessary for regulatory approval. What is recognized now is that the way in which these medications may achieve the $\mathrm{HbA1c}$ improvements as well as their non-glycemic effects are important, particularly cardiovascular safety.

Also important to consider is that a significant proportion of those living with diabetes may be from countries where cost of the newer medications may be prohibitive for a number of reasons. It is fortuitous that the updated American Diabetes Association (ADA)/European Association for the Study of Diabetes (EASD) guidelines (2018) [1] recognize this large subgroup of patients, in addition to emphasizing the need for patient-centered care, education and engagement of the patient as well as health literacy and lifestyle changes at each stage.
The "new kids on the block", although now not so "new", include the sodium/glucose cotransporter-2 (SGLT-2) inhibitors, dipeptidyl peptidase IV (DPP-IV) inhibitors, and glucagon-like peptide 1 receptor agonists (GLP-1 RA).

Recent cardiovascular outcome trials (CVOTs) have demonstrated benefits of SGLT-2 inhibitors in decreasing hospitalization for heart failure in populations with and without existing cardiovascular disease (CVD), with less consistent effects on other outcomes such as cardiovascular (CV) death. The EMPA-REG study [2] was the first of these studies to be presented. It demonstrated that, in a population with established CVD, those randomized to empagliflozin had a decreased risk of $\mathrm{CV}$ death. The CANVAS [3] and DECLARETIMI [4] studies included populations with and without established CVD, the former showing superiority of the drug on MACE outcomes (major adverse cardiovascular events, a composite of death, myocardial infarction, or repeat coronary revascularization of the target lesion), whilst the latter study demonstrated that the use of dapagliflozin was non-inferior for MACE outcomes and decreased the risk of renal disease, a feature also of the use of SGLT-2 inhibitors in the other two studies.

These CV and renal benefits were the first seen for any class of oral anti-hyperglycemic agents. However, these benefits or "ups" need to be balanced with potential side effects.
St Vincent'

Hospital, Sydney

2 Conjoint Associate

Professor, University

of New South Wales

3 Clinical Associate

Professor, University of Sydney

${ }^{4}$ Visiting Scientist, Garvan Institute of Medical

Research, New South

Wales, Australia

Endocrinology Registrar,

Nepean Hospital,

Sydney, New South

Wales, Australia
Corresponding author Roger Chen

roger.chen@sydney.edu.au

Received: 18 March 2019 Accepted: 18 March 2019 Published: 3 April 2019 
These include the well-known risk of mycotic genitourinary infections, hypotension and increased urination, as well as a rarer but recognized association with a new entity of euglycemic diabetic ketoacidosis [5]. A tantalizing signal of increased lower limb amputations and small bone fractures was also seen in the CANVAS study [3]. These similarities and differences have sparked considerable debate as to whether there are within class differences which might account for these disparities and whether differences in SGLT-1 and SGLT-2 receptor affinity are relevant [6]. However, it should always be noted that studies involved different populations and that direct comparisons may be fraught with limitations. However, these "downs" seem to be relatively small in comparison with the "ups" which include CV as well as renal benefits. Nevertheless, the physician will need to be familiar with these potential side effects, to have a checklist when prescribing and to ensure that these side effects and precautions are explained to the patient. The results of dedicated studies in renal impairment such as CREDENCE as well as those addressing heart failure specifically including EMPEROR-Preserved and EMPEROR-Reduced will strengthen our knowledge of where and when these drugs might fit in [7].

The DPP IV inhibitors seem to be overall neutral in their effects of CV risk, although there was an unexplained increase in hospitalizations for heart failure in those subjects taking the active drug in the SAVOR-TIMI study [8]. However, overall these medications appear to be moderate in their potential glucose-lowering effects and in general safe and simple to prescribe, particularly when combined with metformin, especially in the older patient. In some cases, dose reductions need to be made where there is renal dysfunction.

There are a variety of GLP-1 RA which can be divided into short-acting, where daily or twice daily administration is required, to longer-acting agents, which can be administered daily to weekly. Longer-acting agents, which may be able to be given monthly or even 6 monthly, are in development. Results of the LEADER [9] and SUSTAIN-6 [10] study indicate potential CV benefits of liraglutide and semaglutide when administered to a population at high risk of CVD whilst others have been non-inferior for $\mathrm{CV}$ outcomes (ELIXA - lixisenatide and EXSCEL-bydureon) $[11,12]$. The yet unpub- lished topline results of the REWIND study (dulaglutide) indicate $\mathrm{CV}$ benefits in those receiving dulaglutide. The other benefits of this class are that weight loss can occur together with improvements in blood pressure and other metabolic parameters. However, ways in which to predict who will respond best remain elusive and emphasize the need always to individualize therapy.

The "old dogs" such as metformin, sulphonylureas, and thiazolidinediones (TZDs) cannot be forgotten as these are often either prescribed as first-line medications (metformin) or are affordable in many parts of the world in comparison to the newer agents and, as such, are the only medications which are available for use in these countries. Sulphonylureas are potent glucose-lowering agents, but carry an increased risk of hypoglycemia compared with all other noninsulin agents. The results of the CAROLINA study, in which linagliptin is compared with glimeperide, will be presented at the American Diabetes Association Scientific Sessions in 2019 and will potentially provide important data regarding the safety of sulphonylureas.

The CV safety of pioglitazone was demonstrated in the PROactive study [13]. However, this drug class is associated with weight gain, which many patients do not consider favorable, as well as an increased risk of heart failure when combined with insulin [14]. Nevertheless, when used in low dose early in combination with metformin and exenatide, the authors of the EDICT study have demonstrated sustained effects on HbA1c with low rates of hypoglycaemia [15].

One can see from the above that the prescriber now has a variety of classes of medications to choose from; or an "agony of choice". Each class has its "ups and downs" and there may be within class differences, particularly when considering the newer agents. Guidelines are important, but the medication choice ultimately results from the physician and patient interaction. Every "up" and "down" influences the choice of pharmacotherapy, and published benefits such as weight loss may not be perceived by some patients as an advantage. CVOTs and registration studies indicate to the physician what can happen (good and bad) over the defined length of the study. However, what eludes us at this time is how to determine in which person a drug will be effective or ineffective, hence the need for personalized medicine. 
Funding

This article has been published without the support of sponsors.

Conflicts of interests

Roger Chen reports personal fees as speaker in educational meetings/adviser from Novo Nordisk, Sanofi, Eli Lilly, Boehringer Ingelheim, Astra Zeneca and MSD outside the submitted work. Ashish Munsif declare he has no competing financial interests concerning the topics of this article.

\section{REFERENCES}

1. Davies MJ, D'Alessio DA, Fradkin J, et al. Management of hyperglycaemia in type 2 diabetes, 2018. A consensus report by the American Diabetes Association (ADA) and the European Association for the Study of Diabetes (EASD). Diabetologia 2018; 61: 2461-98; https://doi. org/10.1007/s00125-018-4729-5

2. Zinman B, Wanner C, Lachin JM, et al.; EMPA-REG OUTCOME Investigators. Empagliflozin, Cardiovascular Outcomes, and Mortality in Type 2 Diabetes. $N$ Engl J Med 2015; 373: 2117-28; https://doi.org/10.1056/NEJMoa1504720

3. Neal B, Perkovic V, Matthews DR. Canagliflozin and Cardiovascular and Renal Events in Type 2 Diabetes. N Engl J Med 2017; 377: 644-57; https://doi.org/10.1056/NEJMc1712572

4. Wiviott SD, Raz I, Bonaca MP, et al.; DECLARE-TIMI 58 Investigators. Dapagliflozin and Cardiovascular Outcomes in Type 2 Diabetes. N Engl J Med 2019; 380: 347-57; https://doi. org/10.1056/NEJMoa1812389

5. Hamblin PS, Wong R, Ekinci EI, et al. SGLT2 Inhibitors Increase the Risk of Diabetic Ketoacidosis Developing in the Community and During Hospital Admission.J Clin Endocrinol Metab 2019 Mar 5. pii: jc.2019-00139; https://doi.org/10.1210/jc.2019-00139

6. Wu JH, Foote $\mathrm{C}$, Blomster J, et al. Effects of sodium-glucose cotransporter-2 inhibitors on cardiovascular events, death, and major safety outcomes in adults with type 2 diabetes: a systematic review and meta-analysis. Lancet Diabetes Endocrinol 2016; 4: 411-9; https://doi. org/10.1016/S2213-8587(16)00052-8

7. Jardine MJ, Mahaffey KW, Neal B, et al.; CREDENCE study investigators. The Canagliflozin and Renal Endpoints in Diabetes with Established Nephropathy Clinical Evaluation (CREDENCE) Study Rationale, Design, and Baseline Characteristics. Am J Nephrol 2017; 46: 462-72; https://doi.org/10.1159/000484633

8. Scirica BM, Bhatt DL, Braunwald E, et al.; SAVOR-TIMI 53 Steering Committee and Investigators. Saxagliptin and cardiovascular outcomes in patients with type 2 diabetes mellitus. N Engl J Med 2013; 369: 1317-26; https://doi.org/10.1056/NEJMoa1307684

9. Marso SP, Daniels GH, Brown-Frandsen K, et al.; LEADER Steering Committee; LEADER Trial Investigators. Liraglutide and Cardiovascular Outcomes in Type 2 Diabetes. N Engl J Med 2016; 375: 311-22; https://doi.org/10.1056/NEJMoa1603827

10. Marso SP, Bain SC, Consoli A, et al.; SUSTAIN-6 Investigators. Semaglutide and Cardiovascular Outcomes in Patients with Type 2 Diabetes. N Engl J Med 2016; 375: 1834-44; https://doi. org/10.1056/NEJMoa1607141

11. Pfeffer MA, Claggett B, Diaz R, et al.; ELIXA Investigators. Lixisenatide in Patients with Type 2 Diabetes and Acute Coronary Syndrome. N Engl J Med 2015; 373: 2247-57; https:// doi.org/10.1056/NEJMoa1509225

12. Holman RR, Bethel MA, Mentz RJ, et al.; EXSCEL Study Group. Effects of Once-Weekly Exenatide on Cardiovascular Outcomes in Type 2 Diabetes. N Engl J Med 2017; 377: 122839; https://doi.org/10.1056/NEJMoa1612917

13. Dormandy JA, Charbonnel B, Eckland DJ, et al.; PROactive Investigators. Secondary prevention of macrovascular events in patients with type 2 diabetes in the PROactive Study (PROspective pioglitAzone Clinical Trial In macroVascular Events): a randomised controlled trial. Lancet 2005; 366: 1279-89; https://doi.org/10.1016/S0140-6736(05)67528-9

14. Graham DJ, Ouellet-Hellstrom R, MaCurdy TE, et al. Risk of acute myocardial infarction, stroke, heart failure, and death in elderly Medicare patients treated with rosiglitazone or pioglitazone. JAMA 2010; 304: 411-8; https://doi.org/10.1001/jama.2010.920

15. Abdul-Ghani MA, Puckett $\mathrm{C}$, Triplitt $\mathrm{C}$, et al. Initial combination therapy with metformin, pioglitazone and exenatide is more effective than sequential add-on therapy in subjects with new-onset diabetes. Results from the Efficacy and Durability of Initial Combination Therapy for Type 2 Diabetes (EDICT): a randomized trial. Diabetes Obes Metab 2015; 17: 268-75; https://doi.org/10.1111/dom.12417 